\title{
lodine Catalyzed Synthesis of Substituted Furans and Pyrans: Reaction Scope and Mechanistic Insights
}

\author{
Domenic P. Pace, ${ }^{\dagger}$ Raphaël Robidas,${ }^{\S}$ Uyen P. N. Tran, ${ }^{\dagger \perp}$ Claude Y. Legault, $* \S$ Thanh V. Nguyen*† \\ ${ }^{\dagger}$ School of Chemistry, University of New South Wales, Sydney, Australia \\ $\S$ Department of Chemistry, Centre in Green Chemistry and Catalysis, Université de Sherbrooke, Sherbrooke, Canada \\ ${ }^{\perp}$ Hoan Hao Research Institute of Biotechnology Applications, Van Hien University, Ho Chi Minh City, Viet Nam \\ Supporting Information Placeholder
}

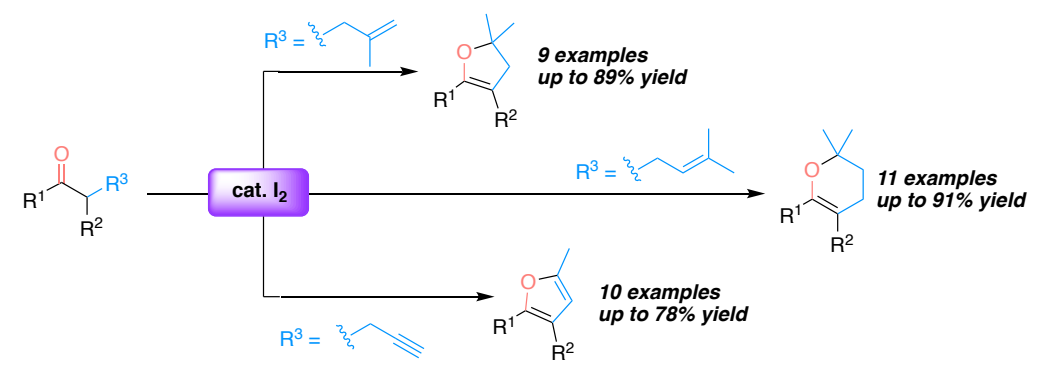

\begin{abstract}
Substituted pyrans and furans are core structures found in a wide variety of natural products and biologically-active compounds. Herein, we report a practical and mild catalytic method for the synthesis of substituted pyrans and furans using molecular iodine, a simple and inexpensive catalyst. The method described herein is performed in solvent-free conditions in ambient temperature and atmosphere, thus offering a facile and green alternative to currently available reaction protocols. A combination of experimental studies and high-level DFT calculations revealed interesting mechanistic insights of this seemingly simple reaction.
\end{abstract}

Molecular iodine, an environmentally-friendly and inexpensive commodity chemical, has long been known not only as a versatile reagent but also an efficient catalyst to promote a wide variety of chemical transformations. ${ }^{1}$ Being the largest non-radioactive element in group 7 of the periodic table, iodine is also the least electronegative and the most polarizable halogen. ${ }^{1 \mathrm{c}, 2}$ These properties lead to its several oxidation states in several classes of synthetically valuable organoiodines. ${ }^{3}$ In elemental form, its ability to interact with oxygen-containing functional groups has been exploited to design efficient iodine-catalyzed organic reactions such as Michael, aldol, esterification and a range of cycloaddition reactions. ${ }^{1}$ Realization of the full catalytic potential of molecular iodine has consistently been a topic of interest in the past decades. ${ }^{\text {dd }}$

Recently, our group discovered another intriguing catalytic activity of molecular iodine in promoting carbonyl-olefin metathesis. ${ }^{4}$ However, in the course of that study we discovered an unexpected 6-endo-trig cyclization reaction of a $\gamma$-alkenyl ketone to produce a 3,4-dihydro-2H-pyran derivative (Scheme 1). Pyrans and their oxygen-containing heterocyclic siblings, furans, are important structural elements of many natural products $^{5}$ and pharmaceuticals, ${ }^{6}$ as well as valuable reaction precursors or intermediates in organic synthesis. ${ }^{7}$ There are several synthetic strategies which yield pyrans and furans bearing different substitution patterns. , $^{7-8}$ One frequently used method involves cyclization or cycloaddition reactions of unsaturated carbonyl compounds using transition-metal catalysts ${ }^{8 \mathrm{e}}$ such as $\mathrm{Hg},{ }^{9} \mathrm{Au},{ }^{10} \mathrm{Pd},{ }^{11} \mathrm{Ag},{ }^{12} \mathrm{Co},{ }^{13} \mathrm{Zn},{ }^{14} \mathrm{Cu},{ }^{15} \mathrm{Rd},{ }^{16} \mathrm{Pt},{ }^{17}$ and other metals such as $\mathrm{Ca},{ }^{18} \mathrm{Ga},{ }^{19} \mathrm{Sc}^{20}$ and $\mathrm{Bi}^{21}$

In $2008 \mathrm{Zhan}$ and co-workers reported an interesting $\mathrm{FeCl}_{3}$-catalyzed one-pot propargylation and cycloisomerisation of 1,3dicarbonyl compounds to form tetrasubstituted furans. ${ }^{22}$ Other iron catalysts were also found to promote different synthetic approaches to furans and pyrans. ${ }^{23}$ Schindler and co-workers subsequently identified $\mathrm{FeCl}_{3}$ as an efficient Lewis acid-based catalyst for the formation of a range of 3,4-dihydro-2H-pyrans ${ }^{24}$ and 3-carboxy-2,5-disubstituted furans. ${ }^{25}$ This simple catalyst also proves to be an efficient promoter for the intramolecular carbonyl-olefin metathesis reaction in a series of elegant works by the Schindler group. ${ }^{26}$ The similarity in catalytic activity between $\mathrm{FeCl}_{3}$ and iodine, demonstrated by these studies and our earlier work (Scheme 1), ${ }^{4}$ prompted us to investigate the possibility of using molecular iodine as catalyst for the synthesis of a broader scope of pyrans and furans.

$\mathrm{I}_{2}$ has been used previously in super-stoichiometric amounts to obtain iodofurans via the iodoenoletherification of 2-alkenyl substituted 1,3-dicarbonyl compounds or iodocyclization of ynenyl acetate. ${ }^{27}$ However, extraneous deiodination and acidcatalyzed isomerisations reactions were required to obtain the non-iodinated furan products. Notably, an $\mathrm{I}_{2}-\mathrm{PPh}_{3}$ catalytic 


$$
\text { (ref. 28) }
$$
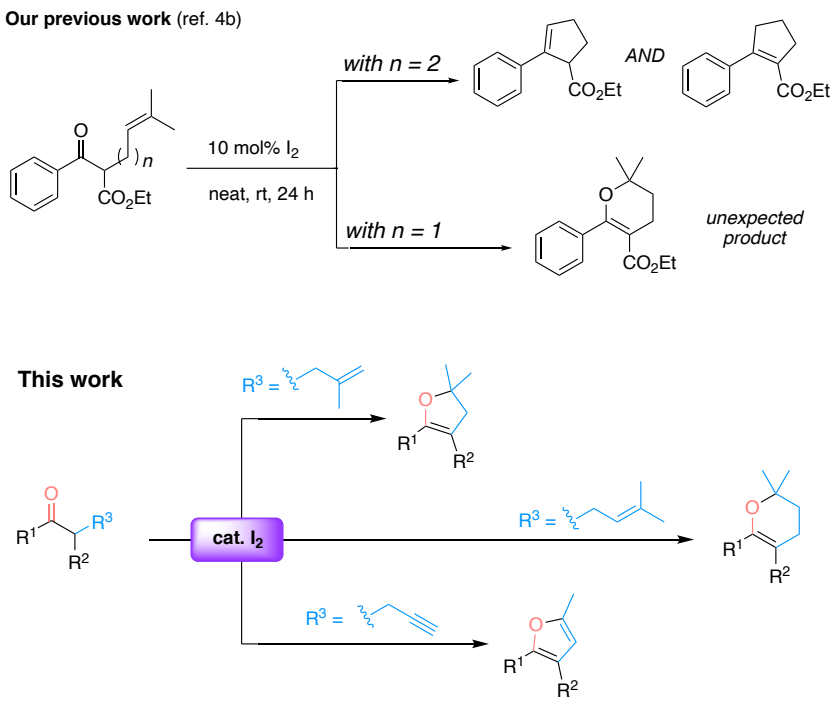

Scheme 1. Iodine-catalyzed reactions

system was previously reported to promote the synthesis of furan-type spiro enol ether derivatives from unsaturated $\beta$-ketoesters. ${ }^{17}$ However, these examples are scarce and non-systematic. Herein, we demonstrate that $I_{2}$ catalyst can efficiently promote the cyclisation of a broad range of $\alpha$-allylated or $\alpha$-propargylated carbonyl substrates to form a wide range of substituted pyrans and furans under mild reaction conditions with excellent outcomes.

We started our investigation by optimizing the serendipitous reaction with substrate 1a (Table 1) to form product 2a. Optimization studies (see page $\mathrm{S} 3$ in the SI) ${ }^{28}$ confirmed that the reactions could be performed efficiently under ambient atmosphere and temperature with solvent-free conditions using $10 \mathrm{~mol} \% \mathrm{I}_{2}$ catalyst to afford product 2a in $89 \%$ yield. These optimal conditions were amenable to a range of other $\alpha$-prenylated aryl or alkyl ketones (Table 1) to form corresponding 3,4-dihydro- $2 \mathrm{H}$ pyrans in good to excellent yields for both alkyl and aryl ketones. The protocol tolerates a good range of functional groups, as expected from the benign nature of iodine. Interestingly, the reaction proceeded smoothly with electron-deficient aryl groups (entries 2 to 4 , Table 1), whilst only trace amount of the desired pyran was observed with an electron-rich aryl group (entry 5). It is possible that an electronic aromatic iodination side reaction rendered the iodine catalyst inactive. Similar diminishing effect also occurred with the bulky tert-butyl substituent (entry 13, Table 1), presumably due to its steric hindrance. When there was an aryl group also at the $\alpha$-position, the reaction led to a mixture of the desired pyran $\mathbf{2 h}$ as well as the tetrahydronaphthalene derivative $\mathbf{2 h}$ ' as the minor product (entry 8 , Table 1). Compound $\mathbf{2 h}$ ' was presumably the outcome of the Friedel-Crafts type alkylation reaction on the side-chain phenyl group, as was also previously observed with $\mathrm{FeCl}_{3}$ catalyst by the Schindler group. ${ }^{29}$ With diketone $\mathbf{1 g}$ (entry 7, Table 1) where there are two carbonyl groups competing for the reaction, it was interesting to see that the cyclization preferred to occur on the aliphatic ketone.
Table 1. Substrate scope for 3,4-dihydro-2H-pyrans ${ }^{a}$<smiles>[R7]C1=C(C)OC(C)(C)CC1</smiles>

(2)

${ }^{a}$ Reaction conditions: $\beta$-ketoester $(0.5 \mathrm{mmol})$ and iodine $(0.05$ mmol) at rt for 24 h. ${ }^{b}$ Yield of the isolated product. ${ }^{c}$ Same conditions but with $20 \mathrm{~mol} \% \mathrm{I}_{2} .{ }^{d}$ Same conditions but at $50{ }^{\circ} \mathrm{C}$. See the experimental Supporting Information for more details. 
Scheme 2. Synthesis of 3-carboxy-2,5-disubstituted furans ${ }^{a}$
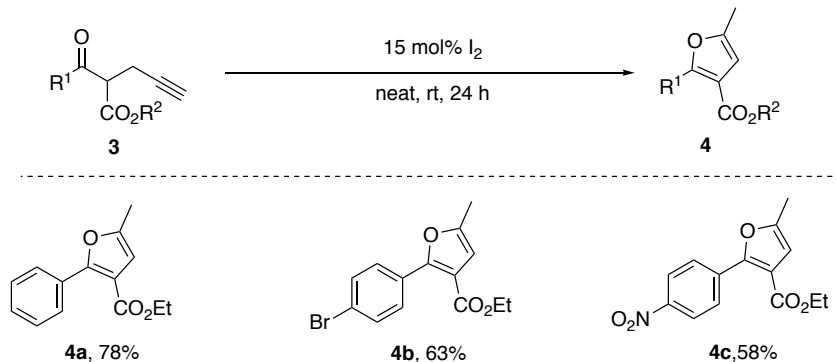

$4 a, 78 \%$

$4 b, 63 \%$

$4 c, 58 \%$

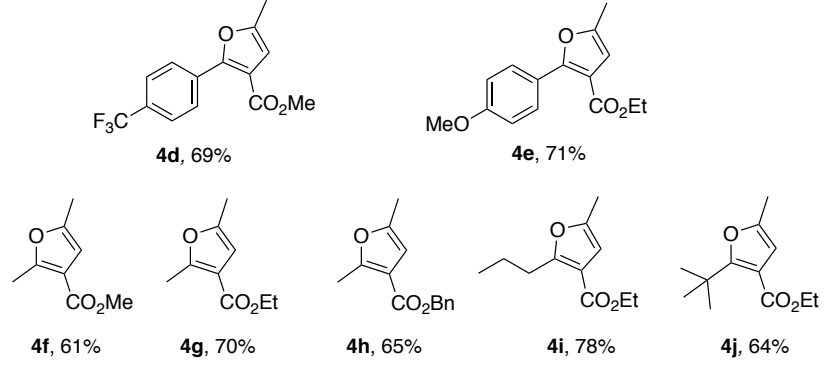

${ }^{a}$ Reaction conditions: $3(0.5 \mathrm{mmol})$ and iodine $(0.075 \mathrm{mmol})$ at $\mathrm{rt}$ for $24 \mathrm{~h}$. Yield of the isolated product, see the Supporting Information for more details.

We then turned our attention to investigate the catalytic activity of $I_{2}$ in the synthesis of 3-carboxy-2,5-disubstituted furans from the $\alpha$-propargyl- $\beta$-ketoester substrates (Scheme 2 ). Similar optimization studies proved that $15 \mathrm{~mol} \%$ iodine catalyst is the most efficient loading for this reaction. ${ }^{28}$ The reaction worked relatively well on the ten substrates we studied. Overall, yields were lower than the synthesis of pyrans in Table 1, which is most likely due to the formation of unwanted byproducts owing to the inherent reactivity of alkynes. However, substrates with electron-rich aryl group (3e) or bulky alkyl group (3j) still reacted smoothly to give the products in good yields.

Similarly, the synthesis of 3-carboxy-2,2,5-trisubstituted-4,5dihydrofurans from analogous alkenyl precursors also

\section{Scheme 3. 3-carboxy-2,2,5-trisubstituted-4,5-dihydrofu- rans $^{a}$}
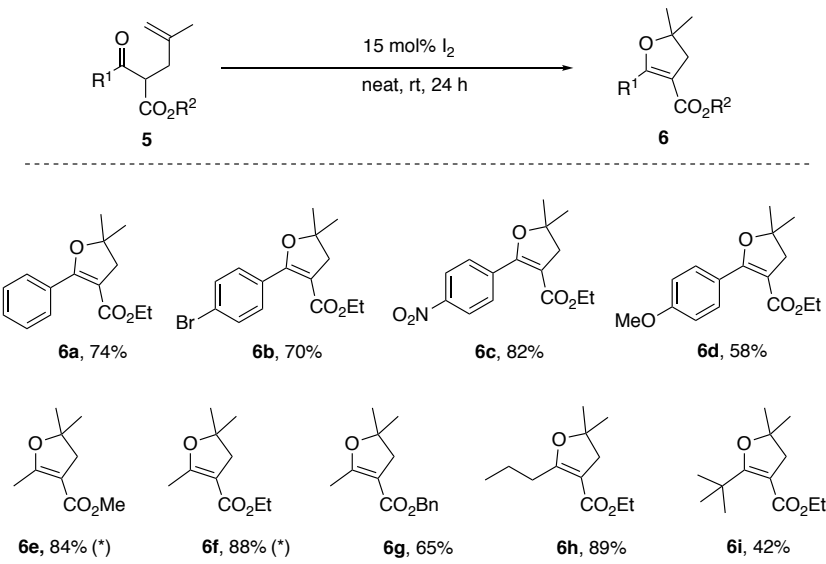

${ }^{a}$ Reaction conditions: $5(0.5 \mathrm{mmol})$ and iodine $(0.075 \mathrm{mmol})$ at $\mathrm{rt}$ for $24 \mathrm{~h}$. Yield of the isolated product. $(*)=$ yield determined by ${ }^{1} \mathrm{H}$ NMR using 1,3,5-triisopropylbenzene as an internal standard due to the volatility of the products, see the Supporting Information for more details. proceeded in good to excellent yield (Scheme 3). Curiously, there was a decrease in yields, in comparison to other substrates, for the methoxyarene- and tert-butyl-containing products $\mathbf{6 d}$ and 6i (Scheme 3), which is presumably related to the same trend in Table 1. Overall, molecular iodine proves to be an efficient catalyst for the cyclization reaction of alkenyl or alkynyl carbonyl substrates to produce pyran and furan derivatives.

Based on our previous studies with iodine-catalyzed carbonylolefin metathesis reaction, ${ }^{4 \mathrm{~b}, 4 \mathrm{c}}$ we suspected that this cyclization reaction could be catalyzed via one of some possible pathways such as a halogen-bonding, Brønsted acid, higher oxidation state iodine, such as iodonium ion, or radical catalysis. ${ }^{1 \mathrm{~d}, 30} \mathrm{We}$ subsequently carried out a range of mechanistic studies to learn more about this system (Table 2). While radical traps such as TEMPO or BHT had diminishing effect on the catalytic activity of $\mathrm{I}_{2}$ (entries 16-17), high energy photo-irradiation (entries 2-3), with the intention to trigger iodine radical formation, significantly reduced reaction efficiency. From both of these two observations, we believe that a radical pathway is unlikely.

The reaction efficiency did not change much when we switched to inert environment (Table 2, entries 5-6), but oxidative or water-enriched environments led to complicated reaction mixtures (entries 7-9). KI did not have any effect on the reaction by itself (entry 10), apart from suppressing the catalytic activity of $\mathrm{I}_{2}$ (entry 11). The use of $a q$. HI as catalyst led to some conversion to the product (entry 12), suggesting that acidic conditions might help the reaction but are not a determining factor.

The positive but non-efficient outcomes of entries 13-14, all favorable conditions for the formation of $\mathrm{I}^{+}$ion, indicating that the iodonium pathway is not the predominant mechanism either. The addition of DMSO or $\mathrm{PPh}_{3}$ (entries 15 and 18), which can coordinate or react with iodine, turned off the reaction. $\mathrm{Hu}-$ ber's bidentate iodoazolium salt also did not promote this reaction, hinting that it might not be triggered by halogen-bonding (entry 19). ${ }^{2}$ All of these studies suggest that the reaction mechanism might involve the intact molecular iodine catalyst.

To be able to further elucidate the reaction mechanism, we subsequently evaluated several different reaction pathways using high level DFT calculations. The geometry optimizations were performed with the M062X density functional, ${ }^{31}$ in combination with the aug-cc-pVTZ ${ }^{32}$ basis set for all atoms except iodine, for which $\mathrm{SDD}^{33}$ and corresponding ECP were used. The basis sets were selected in accord with the recent benchmarking study of Nakajima, Nemoto, and co-workers. ${ }^{34}$ The reactions were carried in neat conditions, but to account for overall effect of the reaction medium, the calculations were performed using SMD implicit solvation model for dichloromethane, which was also a working solvent for this reaction. ${ }^{28}$ The recently corrected radius for iodine atom for SMD calculations was used. ${ }^{35} \mathrm{Sub}-$ strate $\mathbf{1 i}\left(\mathrm{R}^{1}=\mathrm{Me}, \mathrm{R}^{2}=\mathrm{OMe}\right)$ was selected for the study to reduce the degrees of freedom and simplify analysis. The yield of the reaction with this substrate was also representative of the general scope. We first investigated the possible tautomers of $\mathbf{1 i}$ and their coordinating adducts with molecular iodine $\left(\mathrm{I}_{2}\right)$, as depicted in Scheme 4. ${ }^{36}$ As could be expected for a $\beta$-ketoester derivative, the enol tautomer (1i') is easily accessible at room temperature $(\Delta \mathrm{G}=+2.3 \mathrm{~kJ} / \mathrm{mol})$. Interestingly, all of the substrate $\mathrm{I}_{2}$ coordinating adducts are formed exergonically, but a stark thermodynamic preference is found for the association to alkene/enol groups. ${ }^{36}$ The association of $\mathrm{I}_{2}$ to either the ketone or ester carbonyl moiety (Int-A, Int-C) is only slightly stabilizing. 
Table 2. Mechanistic studies ${ }^{a}$

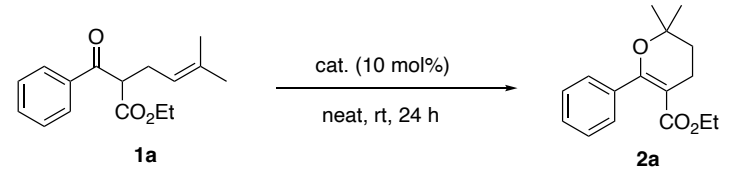

\begin{tabular}{|c|c|c|}
\hline entry & cat. and conditions ${ }^{b}$ & yield $^{c}(\%)$ \\
\hline 1 & $\mathrm{I}_{2}$ (ambient atmosphere, lab light) & 89 \\
\hline 2 & $\mathrm{I}_{2}$ (ambient atmosphere, $365 \mathrm{~nm}$ ) & messy reaction \\
\hline 3 & $\mathrm{I}_{2}$ (ambient atmosphere, blue LED) & 36 \\
\hline 4 & $\mathrm{I}_{2}$ (ambient atmosphere, white LED) & 82 \\
\hline 5 & $\mathrm{I}_{2}$ (under argon, lab light) & 80 \\
\hline 6 & $\mathrm{I}_{2}$ (under argon, in the dark) & 76 \\
\hline 7 & $\mathrm{I}_{2}$ (under $\mathrm{O}_{2}$, lab light) & messy reaction \\
\hline 8 & $\mathrm{I}_{2}(10 \mathrm{~mol} \%)+m \mathrm{CPBA}(20 \mathrm{~mol} \%)$ & messy reaction \\
\hline 9 & $\mathrm{I}_{2}(10 \mathrm{~mol} \%)+$ water $(20 \mathrm{~mol} \%)$ & 10 \\
\hline 10 & KI & n.r. \\
\hline 11 & $\mathrm{I}_{2}(10 \mathrm{~mol} \%)+\mathrm{KI}(20 \mathrm{~mol} \%)$ & 16 \\
\hline 12 & HI (aq. $57 \%$ w/w) & 16 \\
\hline 13 & NIS & 38 \\
\hline 14 & $\mathrm{ICl}$ & 31 \\
\hline 15 & $\mathrm{I}_{2}(10 \mathrm{~mol} \%)+\mathrm{DMSO}(20 \mathrm{~mol} \%)$ & n.r. \\
\hline 16 & $\mathrm{I}_{2}(10 \mathrm{~mol} \%)+$ TEMPO $(20 \mathrm{~mol} \%)$ & messy reaction \\
\hline 17 & $\mathrm{I}_{2}(10 \mathrm{~mol} \%)+$ BHT $(20 \mathrm{~mol} \%)$ & n.r. \\
\hline 18 & $\mathrm{I}_{2}(10 \mathrm{~mol} \%)+\mathrm{PPh}_{3}(10 \mathrm{~mol} \%)$ & 23 \\
\hline 19 & & n.r. \\
\hline 20 & $\mathrm{I}_{2}(10 \mathrm{~mol} \%)+$ TFA $(10 \mathrm{~mol} \%)$ & 68 \\
\hline 21 & $\mathrm{I}_{2}(10 \mathrm{~mol} \%)+\mathrm{NaOAc}(20 \mathrm{~mol} \%)$ & n.r. \\
\hline
\end{tabular}

${ }^{a}$ Reaction conditions: $1 \mathrm{a}(0.5 \mathrm{mmol})$ and catalyst $(0.05 \mathrm{mmol})$ at rt for $24 \mathrm{~h} .{ }^{b}$ Entries $1-7$ used $10 \mathrm{~mol}^{\circ} \mathrm{I}_{2}$; lab light means lighting conditions inside the fumehood ( 4 x $13 \mathrm{~W}$ fluorescent lights); entries 8-21 used ambient atmosphere and lab light again. ${ }^{c}$ Yield determined by ${ }^{1} \mathrm{H}$ NMR using 1,3,5-triisopropylbenzene as an internal standard; messy reactions means there were many unidentified products formed along with less than $10 \%$ conversion to $\mathbf{2 a}$; n.r. means no conversion to product $2 \mathbf{a}$ was observed.

It is interesting to note that the complexation of iodine to the alkene is even more favorable in the enol form than in the keto form (Int-B vs Int-D). Analysis of the structures of Int-B and Int-D suggests that this stabilization could be attributed to additional favorable electrostatic $\mathrm{O}^{\delta \cdots} \mathrm{I}^{\delta+}$ interactions in Int-D (Figure 1). A notable effect of the association of $I_{2}$ to the enol is observed in Int-E, when compared to the structural features of the enol in Int-D. In particular, the enol-carbonyl hydrogen bond in Int-E is greatly shortened (1.57 $\AA$ compare to $1.64 \AA$ in Int-D, Figure 1). ${ }^{36}$ We postulated that the association of $\mathrm{I}_{2}$ significantly increases $\mathrm{O}_{\text {enol- }} \mathrm{H}$ acidity. With this assumption in mind, we investigated a mechanism in which this $\mathrm{O}_{\text {enol }}-\mathrm{H}$ would be sufficiently acidic to protonate the alkene sidechain and lead to the formation of a carbocationic intermediate that would
Scheme 4. Possible substrate $\boldsymbol{I}_{\mathbf{2}}$ adducts (Free energies values reported in $\mathrm{kJ} / \mathrm{mol}$ )

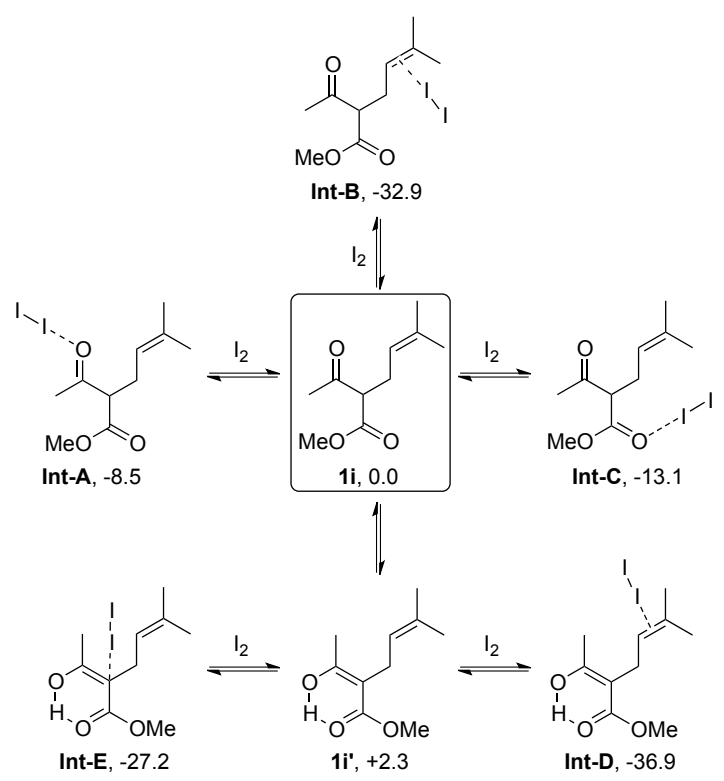

ultimately lead to the desired product. A test reaction with trifluoroacetic acid additive (entry 20, Table 2) led to slightly lower but still good efficiency as compared to the standard conditions. Another test reaction with NaOAc additive (entry 21, Table 2) to scramble any in situ generated Brønsted acidic or carbocationic species gave negative results. Both of these two additional studies support our proposed mechanism. The results of the calculations are illustrated in Scheme 5.
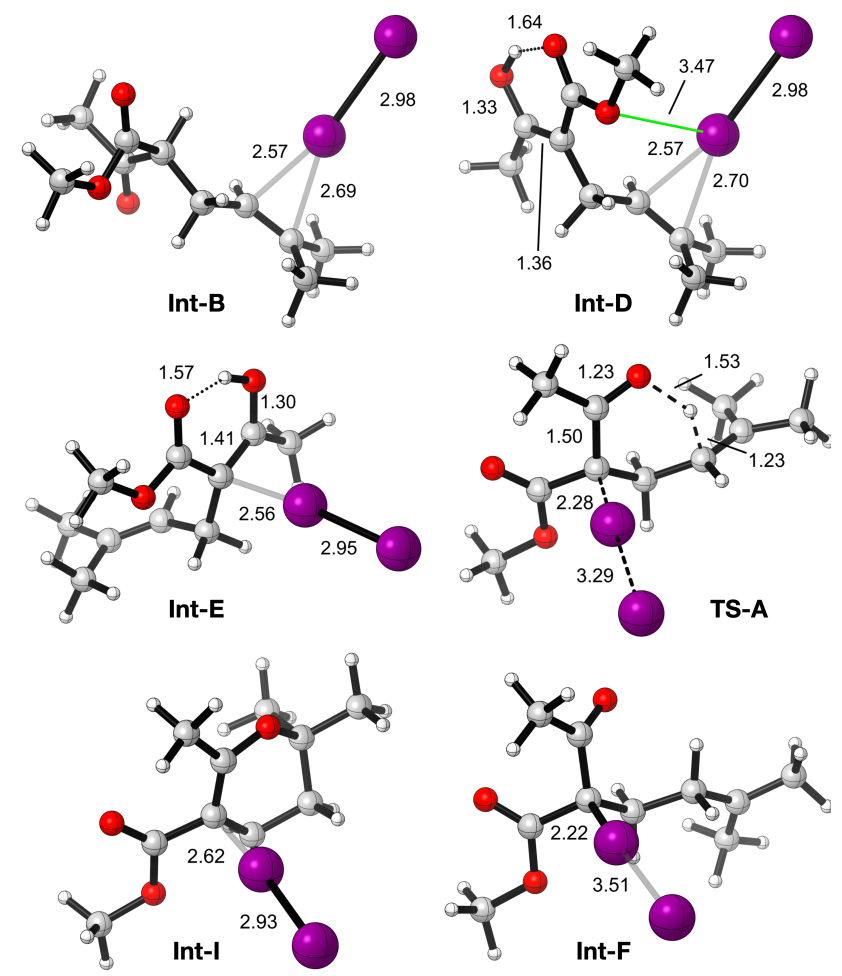

Figure 1. Structures of selected intermediates and transition-state in Schemes 4 and 5 (bond lengths in $\AA$ ). 
Scheme 5. Plausible catalytic cycle supported by DFT calculations (Free energies reported in $\mathrm{kJ} / \mathrm{mol}$ )

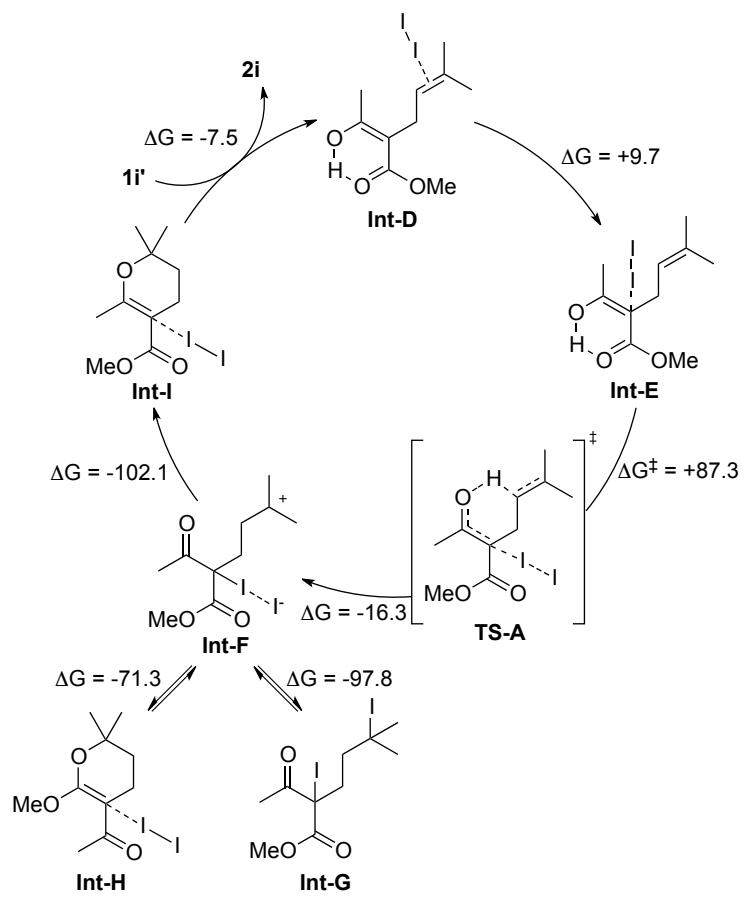

Gratifyingly, the transition state of intramolecular proton transfer (TS-A) did indeed prove to be reasonable $\left(\Delta \mathrm{G}^{\ddagger}=+87.3\right.$ $\mathrm{kJ} / \mathrm{mol}$ from Int-E). Through this process, the carbon-iodine bond shortens from $2.56 \AA$ to $2.22 \AA$, indicating the formation of a fully covalent C-I bond. Accordingly, the iodine-iodine bond lengthens from $2.95 \AA$ to $3.51 \AA$. With respect to the most stable substrate $\boldsymbol{I}_{2}$ adduct (Int-D), TS-A is considered to be the highest transition state in our postulated mechanism on the energy diagram, leading to the key carbocationic intermediate Int-F. In this context, the energetic span of the process is found to be $97 \mathrm{~kJ} / \mathrm{mol}$ (Int-D to TS-A). This value is in very good agreement (deviation below $4 \mathrm{~kJ} / \mathrm{mol}$ ) with the estimated experimental energetic span for substrate $1 i^{36}{ }^{36}$ Furthermore, kinetic data is in accord with the kinetic model describing the proposed mechanism (page S6-S8 in the computational SI). ${ }^{36}$

The resulting carbocationic intermediate (Int-F) is expected to react in mostly barrierless processes with the various nucleophilic sites on the intermediate, leading to three possible intermediates, Int-G, Int-H, and Int-I. Not surprisingly, all three pathways are found to be exergonic, but a deeper look into the thermodynamics provides interesting insights. Collapse of the iodide anion onto the carbocation results in the formation of diiodoalkyl intermediate Int-G and a stabilization energy of 97.8 $\mathrm{kJ} / \mathrm{mol}$. Interestingly, this value is almost identical to the energetic span leading to formation of Int-F (vide supra). It is thus foreseeable that either proton transfer (TS-A), or heterolysis of Int-G, to generate Int-F, is rate-determining. It should be noted that no transition state for the $S_{\mathrm{N}} 1$-like $\mathrm{S}_{\mathrm{N}} 2$ reaction of Int-G to form Int-I was found. The reaction thus more likely proceeds by a $\mathrm{S}_{\mathrm{N}} 1$ mechanism.

Although exergonic, attack of the ester group on the carbocation, resulting in Int-H, provides a stabilization of only $71.3 \mathrm{~kJ} / \mathrm{mol}$, and is expected to be reversible. Cyclization by the attack of the ketone group to the carbocation centre, to form IntI, is much more exergonic $(\Delta \mathrm{G}=-102.1 \mathrm{~kJ} / \mathrm{mol})$, and is considered to be irreversible at room temperature. Following cyclization, I-I bond is regenerated in Int-I; the latter can be considered to be $\mathbf{2} \mathbf{i} \cdot \mathbf{I}_{\mathbf{2}}$ adduct. As a final step, to close the catalytic cycle, iodine must be transferred to a new substrate molecule. This step was found to be exergonic with respect to $\mathbf{1 i}$ ', and even reference $1 \mathbf{i}(\Delta \mathrm{G}=-5.2 \mathrm{~kJ} / \mathrm{mol})$. It can thus be concluded that the there is no significant inhibition of the reaction by the product. Product $2 \mathbf{i}$ is more stable by $26.6 \mathrm{~kJ} / \mathrm{mol}$ with respect to substrate $\mathbf{1 i}$, confirming that the overall reaction is exergonic. It is interesting to note that the same comparison of $1 \mathbf{i}$ with the product resulting from the cyclization of the ester group (2i', derived from Int-H) is less stable than $\mathbf{1 i}$ by $22.2 \mathrm{~kJ} / \mathrm{mol}$. This supports the fact that, despite the possible formation of this alternative product, it is globally endergonic and reversible.

In conclusion, we have developed a new practical protocol for the synthesis of 3,4-dihydro-2H-pyrans, 3-carboxy-2,2,5-trisubstituted-4,5-dihydrofurans and 3-carboxy-2,5-disubstituted furans using molecular iodine as a catalyst under mild reaction conditions. The method tolerates a large range of functional groups and offers an easy, green alternative to currently available reaction protocols to access pyran and furan derivatives. A combination of experimental studies and high-level DFT calculations revealed interesting mechanistic insights of this reaction. They strongly support that the association of iodine to the enol tautomer of the substrate enhances its acidity. This key intermediate is a sufficiently strong Brønsted acid to promote protonation of the alkene and formation of a key carbocation, from which only the formation of the desired product is irreversible.

\section{ASSOCIATED CONTENT}

\section{Supporting Information}

The Supporting Information is available free of charge on the ACS Publications website: Experimental details and spectroscopic data for all products, full Gaussian reference, Cartesian coordinates, electronic and free energies.

\section{AUTHOR INFORMATION}

\section{Corresponding Author}

*E-mail: t.v.nguyen@unsw.edu.au

*E-mail: claude.legault@usherbrooke.ca

\section{Author Contributions}

The manuscript was written through contributions of all authors. All authors have given approval to the final version of the manuscript.

\section{ACKNOWLEDGMENT}

The authors thank the Australian Research Council (grants FT180100260 and DP200100063 to TVN) for financial support. DPP is grateful for his RTP PhD scholarship. Computational resources were provided by Calcul Québec and Compute Canada. RR is grateful to NSERC for a Canada Graduate Scholarship (CGS M).

\section{REFERENCES}

1. (a) Jereb, M.; Vražič, D.; Zupan, M., Iodine-catalyzed transformation of molecules containing oxygen functional groups. Tetrahedron 2011, 67 (7), 1355-1387; (b) Ren, Y.-M.; Cai, C.; Yang, R.C., Molecular iodine-catalyzed multicomponent reactions: an efficient 
catalyst for organic synthesis. RSC Adv. 2013, 3 (20), 7182; (c) Yusubov, M. S.; Zhdankin, V. V., Iodine catalysis: A green alternative to transition metals in organic chemistry and technology. Resource-Efficient Technologies 2015, 1 (1), 49-67; (d) Breugst, M.; von der Heiden, D. Mechanisms in Iodine Catalysis. Chem. Eur. J. 2018, 24 (37), 9187-9199. 2. Sutar, R. L.; Huber, S. M., Catalysis of Organic Reactions through Halogen Bonding. ACS Catal. 2019, 9 (10), 9622-9639.

3. (a) Wang, L.; Zhou, X.; Fredimoses, M.; Liao, S.; Liu, Y., Naturally occurring organoiodines. RSC Adv. 2014, 4 (101), 57350-57376; (b) Grelier, G.; Darses, B.; Dauban, P., Hypervalent organoiodine compounds: from reagents to valuable building blocks in synthesis. Beilstein J. Org. Chem. 2018, 14, 1508-1528.

4. (a) Tran, U. P. N.; Oss, G.; Pace, D. P.; Ho, J.; Nguyen, T. V., Tropylium-promoted carbonyl-olefin metathesis reactions. Chem. Sci 2018, 9 (23), 5145-5151; (b) Oss, G.; Nguyen, T. V., Iodonium-Catalyzed Carbonyl-Olefin Metathesis Reactions. Synlett 2019, 30 (17), 1966-1970; (c) Tran, U. P. N.; Oss, G.; Breugst, M.; Detmar, E.; Pace, D. P.; Liyanto, K.; Nguyen, T. V., Carbonyl-Olefin Metathesis Catalyzed by Molecular Iodine. ACS Catal. 2019, 9 (2), 912-919.

5. (a) Furan and Its Derivatives. In Heterocycles in Natural Product Synthesis, pp 97-152; (b) Pyran and Its Derivatives. In Heterocycles in Natural Product Synthesis, pp 153-186; (c) Nasir, N. M.; Ermanis, K.; Clarke, P. A., Strategies for the construction of tetrahydropyran rings in the synthesis of natural products. Org. Biomol. Chem. 2014, 12 (21), 3323-3335; (d) Jacques, R.; Pal, R.; Parker, N. A.; Sear, C. E.; Smith, P. W.; Ribaucourt, A.; Hodgson, D. M., Recent applications in natural product synthesis of dihydrofuran and -pyran formation by ring-closing alkene metathesis. Org. Biomol. Chem. 2016, 14 (25), 5875-5893; (e) Vetica, F.; Chauhan, P.; Dochain, S.; Enders, D. Asymmetric organocatalytic methods for the synthesis of tetrahydropyrans and their application in total synthesis. Chem. Soc. Rev. 2017, 46 (6), 16611674.

6. (a) Fravel, B. W., 7.09 - Pyrans and their Benzo Derivatives: Applications. In Comprehensive Heterocyclic Chemistry III, Katritzky, A. R.; Ramsden, C. A.; Scriven, E. F. V.; Taylor, R. J. K., Eds. Elsevier: Oxford, 2008; pp 701-726; (b) Keay, B. A.; Hopkins, J. M.; Dibble, P. W., 3.08 - Furans and their Benzo Derivatives: Applications. In Comprehensive Heterocyclic Chemistry III, Katritzky, A. R.; Ramsden, C. A.; Scriven, E. F. V.; Taylor, R. J. K., Eds. Elsevier: Oxford, 2008; pp 571-623; (c) Baumann, M.; Baxendale, I. R.; Ley, S. V.; Nikbin, N., An overview of the key routes to the best selling 5-membered ring heterocyclic pharmaceuticals. Beilstein J. Org. Chem. 2011, 7, 442-495; (d) Kumar, D.; Sharma, P.; Singh, H.; Nepali, K.; Gupta, G. K.; Jain, S. K.; Ntie-Kang, F., The value of pyrans as anticancer scaffolds in medicinal chemistry. RSC Adv. 2017, 7 (59), 36977-36999.

7. (a) Lipshutz, B. H., Five-membered heteroaromatic rings as intermediates in organic synthesis. Chem. Rev. 1986, 86 (5), 795-819; (b) Pedro, M.; Tomas, T.; Delso, J. I.; Rosa, M., Furan Oxidations in Organic Synthesis: Recent Advances and Applications. Curr. Org. Chem. 2007, 11 (12), 1076-1091; (c) Phillips, A. J.; Henderson, J. A.; Jackson, K. L., 7.07

Pyrans and their Benzo Derivatives: Structure and Reactivity. In Comprehensive Heterocyclic Chemistry III, Katritzky, A. R.; Ramsden, C A.; Scriven, E. F. V.; Taylor, R. J. K., Eds. Elsevier: Oxford, 2008; pp 337418; (d) Wong, H. N. C.; Yeung, K. S.; Yang, Z., 3.06 - Furans and their Benzo Derivatives: Reactivity. In Comprehensive Heterocyclic Chemistry III, Katritzky, A. R.; Ramsden, C. A.; Scriven, E. F. V.; Taylor, R. J. K. Eds. Elsevier: Oxford, 2008; pp 407-496.

8. (a) Donner, C. D.; Gill, M.; Tewierik, L. M., Synthesis of Pyran and Pyranone Natural Products. Molecules 2004, 9 (6), 498-512; (b) Brimble, M. A.; Gibson, J. S.; Sperry, J., 7.08 - Pyrans and their Benzo Derivatives: Synthesis. In Comprehensive Heterocyclic Chemistry III Katritzky, A. R.; Ramsden, C. A.; Scriven, E. F. V.; Taylor, R. J. K., Eds. Elsevier: Oxford, 2008; pp 419-699; (c) Graening, T.; Thrun, F., 3.07 Furans and their Benzo Derivatives: Synthesis. In Comprehensive Heterocyclic Chemistry III, Katritzky, A. R.; Ramsden, C. A.; Scriven, E. F. V.; Taylor, R. J. K., Eds. Elsevier: Oxford, 2008; pp 497-569; (d) Dau Xuan, D., Recent Progress in the Synthesis of Furan. Mini-Rev. Org. Chem 2019, 16 (5), 422-452; (e) Chen, L.; Chen, K.; Zhu, S., Transition-MetalCatalyzed Intramolecular Nucleophilic Addition of Carbonyl Groups to Alkynes. Chem 2018, 4 (6), 1208-1262.

9. Imagawa, H.; Kurisaki, T.; Nishizawa, M., Mercuric TriflateCatalyzed Synthesis of 2-Methylfurans from 1-Alkyn-5-ones. Org. Lett. 2004, 6 (21), 3679-3681.

10. (a) Yao, T.; Zhang, X.; Larock, R. C., AuCl3-Catalyzed Synthesis of Highly Substituted Furans from 2-(1-Alkynyl)-2-alken-1-ones
J. Am. Chem. Soc. 2004, 126 (36), 11164-11165; (b) Suhre, M. H.; Reif, M.; Kirsch, S. F., Gold(I)-Catalyzed Synthesis of Highly Substituted Furans. Org. Lett. 2005, 7 (18), 3925-3927; (c) Liu, Y.; Liu, M.; Guo, S.; Tu, H.; Zhou, Y.; Gao, H., Gold-Catalyzed Highly Efficient Access to $3(2 \mathrm{H})$-Furanones from 2-Oxo-3-butynoates and Related Compounds. Org Lett. 2006, 8 (16), 3445-3448; (d) Zhang, J.; Schmalz, H.-G., Gold(I)Catalyzed Reaction of 1-(1-Alkynyl)-cyclopropyl Ketones with Nucleophiles: A Modular Entry to Highly Substituted Furans. Angew. Chem. Int. Ed. 2006, 45 (40), 6704-6707; (e) Xie, X.; Du, X.; Chen, Y.; Liu, Y., One-Pot Synthesis of Indole-Fused Scaffolds via Gold-Catalyzed Tandem Annulation Reactions of 1,2-Bis(alkynyl)-2-en-1-ones with Indoles. J. Org. Chem. 2011, 76 (21), 9175-9181; (f) Handa, S.; Slaughter, L. M., Enantioselective Alkynylbenzaldehyde Cyclizations Catalyzed by Chiral Gold(I) Acyclic Diaminocarbene Complexes Containing Weak AuArene Interactions. Angew. Chem. Int. Ed. 2012, 51 (12), 2912-2915; (g) Jin, S.; Jiang, C.; Peng, X.; Shan, C.; Cui, S.; Niu, Y.; Liu, Y.; Lan, Y.; Liu, Y.; Cheng, M., Gold(I)-Catalyzed Angle Strain Controlled Strategy to Furopyran Derivatives from Propargyl Vinyl Ethers: Insight into the Regioselectivity of Cycloisomerization. Org. Lett. 2016, 18 (4), 680-683; (h) Liu, Y.; Jin, S.; Wang, Y.; Cui, S.; Peng, X.; Niu, Y.; Du, C.; Cheng, M., A gold(i)-catalyzed substituent-controlled cycloisomerization of propargyl vinyl ethers to multi-substituted furofuran and furopyran derivatives. Chem. Commun. 2016, 52 (37), 6233-6236; (i) Pertschi, R.; Wagner, P.; Ghosh, N.; Gandon, V.; Blond, G., Gold(I)-Catalyzed Synthesis of Furopyrans: Insight into Hetero-Diels-Alder Reactions. Org Lett. 2019, 21 (15), 6084-6088.

11. (a) Xiao, Y.; Zhang, J., Tetrasubstituted Furans by a PdIICatalyzed Three-Component Michael Addition/Cyclization/CrossCoupling Reaction. Angew. Chem. Int. Ed. 2008, 47 (10), 1903-1906; (b) Liu, R.; Zhang, J., Tetrasubstituted Furans by PdII-Catalyzed ThreeComponent Domino Reactions of 2-(1-Alkynyl)-2-alken-1-ones with Nucleophiles and Vinyl Ketones or Acrolein. Chem. Eur. J. 2009, 15 (37), 9303-9306; (c) Xiao, Y.; Zhang, J., Palladium(II)-Catalyzed Domino Reaction of 2-(1-Alkynyl)-2-alken-1-ones with Nucleophiles: Scope, Mechanism and Synthetic Application in the Synthesis of 3,4-Fused Bicyclic Tetrasubstituted Furans. Adv. Synth. Catal. 2009, 351 (4), 617-629; (d) Saito, A.; Enomoto, Y.; Hanzawa, Y., Pd-catalyzed cycloisomerization-allylation of 4-alkynones: synthesis of 5homoallylfuran derivatives. Tetrahedron Lett. 2011, 52 (33), 4299-4302; (e) Yu, S.-Y.; Zhang, H.; Gao, Y.; Mo, L.; Wang, S.; Yao, Z.-J., Asymmetric Cascade Annulation Based on Enantioselective Oxa-DielsAlder Cycloaddition of in Situ Generated Isochromenyliums by Cooperative Binary Catalysis of $\mathrm{Pd}(\mathrm{OAc}) 2$ and (S)-Trip. J. Am. Chem. Soc 2013, 135 (30), 11402-11407; (f) Xia, Y.; Xia, Y.; Ge, R.; Liu, Z.; Xiao Q.; Zhang, Y.; Wang, J., Oxidative Cross-Coupling of Allenyl Ketones and Organoboronic Acids: Expeditious Synthesis of Highly Substituted Furans. Angew. Chem. Int. Ed. 2014, 53 (15), 3917-3921; (g) Schitter, T.; Roy, N. J.; Jones, P. G.; Werz, D. B., Synthesis of Highly Substituted Furans by a Cascade of Formal anti-Carbopalladation/Hydroxylation and Elimination. Org. Lett. 2019, 21 (3), 640-643.

12. (a) Beeler, A. B.; Su, S.; Singleton, C. A.; Porco, J. A., Discovery of Chemical Reactions through Multidimensional Screening. $J$ Am. Chem. Soc. 2007, 129 (5), 1413-1419; (b) Palisse, A.; Kirsch, S. F., Synthesis of Furans through Silver-Catalyzed Propargyl-Claisen Rearrangement Followed by Cyclocondensation. Eur. J. Org. Chem. 2014, 2014 (32), 7095-7098; (c) Arto, T.; Fernández, P.; Fañanás, F. J.; Rodríguez, F., Complex chromene derivatives through a silver-catalysed cascade reaction of simple o-alkynylsalicylaldehydes and alkenes. Chem. Commun. 2016, 52 (91), 13405-13408; (d) Blanc, A.; Bénéteau, V.; Weibel, J.-M.; Pale, P., Silver \& gold-catalyzed routes to furans and benzofurans. Org. Biomol. Chem. 2016, 14 (39), 9184-9205.

13. Roslan, I. I.; Sun, J.; Chuah, G.-K.; Jaenicke, S., Cobalt(II)Catalyzed Electrophilic Alkynylation of 1,3-Dicarbonyl Compounds To Form Polysubstituted Furans via $\pi-\pi$ Activation. Adv. Synth. Catal. 2015, 357 (4), 719-726

14. (a) Sniady, A.; Durham, A.; Morreale, M. S.; Wheeler, K. A.; Dembinski, R., Room Temperature Zinc Chloride-Catalyzed Cycloisomerization of Alk-3-yn-1-ones: Synthesis of Substituted Furans. Org.Lett. 2007, 9 (7), 1175-1178; (b) Yuan, Y.; Tan, H.; Kong, L.; Zheng, Z.; Xu, M.; Huang, J.; Li, Y., Transition-metal-free C-C $\sigma$-bond activation of $\alpha$-aryl ketones and subsequent $\mathrm{Zn}$-catalyzed intramolecular cyclization: synthesis of tetrasubstituted furans. Org. Biomol. Chem. 2019, 17 (10), 2725-2733.

15. (a) Chen, Y.-F.; Wang, H.-F.; Wang, Y.; Luo, Y.-C.; Zhu, H.L.; Xu, P.-F., Base- and Copper-Catalyzed Intramolecular Cyclization for 
the Direct Synthesis of Dihydrofurans. Adv. Synth. Catal. 2010, 352 (7), 1163-1168; (b) Rauniyar, V.; Wang, Z. J.; Burks, H. E.; Toste, F. D., Enantioselective Synthesis of Highly Substituted Furans by a Copper(II)Catalyzed Cycloisomerization-Indole Addition Reaction. J. Am. Chem. Soc. 2011, 133 (22), 8486-8489; (c) Bai, X.; Lv, L.; Li, Z., Coppercatalyzed tandem trifluoromethylation-cyclization of olefinic carbonyls: synthesis of trifluoromethylated 2,3-dihydrofurans and 3,4-dihydropyrans. Org. Chem. Front 2016, 3 (7), 804-808; (d) Miao, T.; Tian, Z.-Y.; He, Y.M.; Chen, F.; Chen, Y.; Yu, Z.-X.; Fan, Q.-H., Asymmetric Hydrogenation of In Situ Generated Isochromenylium Intermediates by Copper/Ruthenium Tandem Catalysis. Angew. Chem. Int. Ed. 2017, 56 (15), 4135-4139.

16. Zhao, W.; Zhang, J., Rhodium-catalyzed tandem nucleophilic addition/bicyclization of diyne-enones with alcohols: a modular entry to 2,3-fused bicyclic furans. Chem. Commun. 2010, 46 (24), 4384-4386.

17. (a) Oh, C. H.; Lee, J. H.; Lee, S. J.; Kim, J. I.; Hong, C. S., Intramolecular Huisgen-Type Cyclization of Platinum-Bound Pyrylium Ions with Alkenes and Subsequent Insertion into a Benzylic C $\mathrm{C}$ Bond Angew. Chem. Int. Ed. 2008, 47 (39), 7505-7507; (b) Ishida, K.; Kusama, H.; Iwasawa, N., Enantioselective Preparation of 8Oxabicyclo[3.2.1] octane Derivatives via Asymmetric [3+2]-Cycloaddition of Platinum-Containing Carbonyl Ylides with Vinyl Ethers. J. Am. Chem. Soc. 2010, 132 (26), 8842-8843; (c) Allegretti, P. A.; Ferreira, E. M., Generation of $\alpha, \beta$-Unsaturated Platinum Carbenes from Homopropargylic Alcohols: Rearrangements to Polysubstituted Furans. Org. Lett. 2011, 13 (21), 5924-5927.

18. Morcillo, S. P.; Leboeuf, D.; Bour, C.; Gandon, V., CalciumCatalyzed Synthesis of Polysubstituted 2-Alkenylfurans from $\beta$-Keto Esters Tethered to Propargyl Alcohols. Chem.Eur.J. 2016, 22 (47), 16974-16978. 19. Wang, H.-S.; Chan, C.-K.; Chang, M.-Y., Ga(OTf)3-mediated synthesis of substituted benzofurans. Tetrahedron 2016, 72 (33), 51325141 .

20. Cao, Z.; Zhang, R.; Meng, X.; Li, H.; Li, J.; Zhu, H.; Chen, G.; Sun, X.; You, J., Sc(OTf)3-catalyzed cyclization of $\alpha$-allylated 1,3dicarbonyls: an efficient access to 2,2-disubstituted 2,3-dihydrofuran derivatives. $R S C$ Adv. 2016, 6 (78), 74582-74585.

21. (a) Nitsch, D.; Bach, T., Bismuth(III) Triflate-Catalyzed Synthesis of Substituted 2-Alkenylfurans. J. Org. Chem. 2014, 79 (13), 6372-6379; (b) Chang, M.-Y.; Cheng, Y.-C.; Lu, Y.-J., Bi(OTf)3-Mediated Cycloisomerization of $\gamma$-Alkynyl Arylketones: Application to the Synthesis of Substituted Furans. Org. Lett. 2015, 17 (5), 1264-1267.

22. Ji, W.-h.; Pan, Y.-m.; Zhao, S.-y.; Zhan, Z.-p., FeCl $<$ sub $>3</$ sub $>$-Catalyzed Propargylation-Cycloisomerization Tandem Reaction: A Facile One-Pot Synthesis of Substituted Furans. Synlett 2008, 2008 (19), 3046-3052.

23. (a) Jiang, H.; Yao, W.; Cao, H.; Huang, H.; Cao, D., IronCatalyzed Domino Process for the Synthesis of $\alpha$-Carbonyl Furan Derivatives via One-Pot Cyclization Reaction. J. Org. Chem. 2010, 75 (15), 5347-5350; (b) Chang, M.-Y.; Chen, Y.-H.; Cheng, Y.-C., Fe(OTf)3mediated synthesis of sulfonyl dihydropyrans. Tetrahedron 2016, 72 (4), 518-524; (c) Lou, J.; Wang, Q.; Wu, K.; Wu, P.; Yu, Z., Iron-Catalyzed Oxidative C-H Functionalization of Internal Olefins for the Synthesis of Tetrasubstituted Furans. Org. Lett. 2017, 19 (12), 3287-3290.

24. Watson, R. B.; Golonka, A. N.; Schindler, C. S., Iron(III) Chloride Catalyzed Formation of 3,4-Dihydro-2 $<\mathrm{i}>\mathrm{H}</ \mathrm{i}>$-pyrans from $\alpha$ Alkylated 1,3-Dicarbonyls. Selective Synthesis of $\alpha$ - and $\beta$-Lapachone. Org. Lett. 2016, 18 (6), 1310-1313.

25. Golonka, A. N.; Schindler, C. S., Iron(III) chloride-catalyzed synthesis of 3-carboxy-2,5-disubstituted furans from $\gamma$-alkynyl aryl- and alkylketones. Tetrahedron 2017, 73 (29), 4109-4114.
26. (a) Ludwig, J. R.; Zimmerman, P. M.; Gianino, J. B.; Schindler, C. S., Iron(III)-catalysed carbonyl-olefin metathesis. Nature 2016, 533 (7603), 374-379; (b) McAtee, C. C.; Riehl, P. S.; Schindler, C. S., Polycyclic Aromatic Hydrocarbons via Iron(III)-Catalyzed CarbonylOlefin Metathesis. J. Am. Chem. Soc. 2017, 139 (8), 2960-2963; (c) Groso, E. J.; Golonka, A. N.; Harding, R. A.; Alexander, B. W.; Sodano, T. M.; Schindler, C. S., 3-Aryl-2,5-Dihydropyrroles via Catalytic Carbonyl-Olefin Metathesis. ACS Catal. 2018, 8 (3), 2006-2011; (d) Watson, R. B.; Schindler, C. S., Iron-Catalyzed Synthesis of Tetrahydronaphthalenes via 3,4-Dihydro-2H-pyran Intermediates. Org. Lett. 2018, 20 (1), 68-71; (e) Albright, H.; Riehl, P. S.; McAtee,C.C.; Reid,J. P.; Ludwig, J. R.; Karp, L. A.; Zimmerman, P. M.; Sigman, M. S.; Schindler, C. S., Catalytic Carbonyl-Olefin Metathesis of Aliphatic Ketones: Iron(III) Homo-Dimers as Lewis Acidic Superelectrophiles. J. Am. Chem. Soc. 2019, 141 (4), 16901700; (f) Riehl, P. S.; Nasrallah, D. J.; Schindler, C. S., Catalytic, transannular carbonyl-olefin metathesis reactions. Chem. Sci.2019, 10 (44), 10267-10274; (g) Rykaczewski, K. A.; Groso, E. J.; Vonesh, H. L.; Gaviria, M. A.; Richardson, A. D.; Zehnder, T. E.; Schindler, C. S., Tetrahydropyridines via FeCl3-Catalyzed Carbonyl-Olefin Metathesis. Org. Lett. 2020, 22 (7), 2844-2848.

27. (a) Antonioletti, R.; Bonadies, F.; Scettri, A., A convenient approach to furan derivatives by I2-induced cyclisation of 2-alkenyl substituted 1,3-dicarbonyl compounds. Tetrahedron Lett. 1988, 29 (39), 4987-4990; (b) Chen, Z.; Huang, G.; Jiang, H.; Huang, H.; Pan, X., Synthesis of 2,5-Disubstituted 3-Iodofurans via Palladium-Catalyzed Coupling and Iodocyclization of Terminal Alkynes. J. Org. Chem. 2011, 76 (4), 1134-1139.

28. See the experimental Supporting Information for more details. 29. Watson, R. B.; Schindler, C. S., Iron-Catalyzed Synthesis of Tetrahydronaphthalenes via 3,4-Dihydro- $2<\mathrm{i}>\mathrm{H}</ \mathrm{i}>$-pyran Intermediates. Org. Lett. 2018, 20 (1), 68-71.

30. (a) Breugst, M.; Detmar, E.; von der Heiden, D., Origin of the Catalytic Effects of Molecular Iodine: A Computational Analysis. ACS Catal. 2016, 6 (5), 3203-3212; (b) von der Heiden, D.; Bozkus, S.; Klussmann, M.; Breugst, M., Reaction Mechanism of Iodine-Catalyzed Michael Additions. J. Org. Chem. 2017, 82 (8), 4037-4043.

31. Zhao, Y.; Truhlar, D. G., The M06 suite of density functionals for main group thermochemistry, thermochemical kinetics, noncovalent interactions, excited states, and transition elements: two new functionals and systematic testing of four M06-class functionals and 12 other functionals. Theor. Chem. Acc. 2008, 120 (1), 215-241.

32. Jr., T. H. D., Gaussian basis sets for use in correlated molecular calculations. I. The atoms boron through neon and hydrogen. J. Chem. Phys. 1989, 90 (2), 1007-1023.

33. Dolg, M.; Wedig, U.; Stoll, H.; Preuss, H., Energy-adjusted ab initio pseudopotentials for the first row transition elements. J. Chem. Phys. 1987, 86 (2), 866-872.

34. Matsumoto, K.; Nakajima, M.; Nemoto, T., Determination of the best functional and basis sets for optimization of the structure of hypervalent iodines and calculation of their first and second bond dissociation enthalpies. J. Phys. Org. Chem. 2019, 32 (8), e3961.

35. Engelage, E.; Schulz, N.; Heinen, F.; Huber, S. M.; Truhlar, D. G.; Cramer, C. J., Refined SMD Parameters for Bromine and Iodine Accurately Model Halogen-Bonding Interactions in Solution. Chem. Eur. J. 2018, 24 (60), 15983-15987.

36. See the computational Supporting Information for more details. 